\title{
BFGAN - building footprint extraction from satellite images
}

\author{
Yilei Shi ${ }^{1}$, Qingyu $\mathrm{Li}^{2}$, Xiao Xiang Zhu ${ }^{2,3}$ \\ ${ }^{1}$ Chair of Remote Sensing Technology (LMF), Technische Universität München (TUM) \\ ${ }^{2}$ Signal Processing in Earth Observation (SIPEO), Technische Universität München (TUM) \\ ${ }^{3}$ Remote Sensing Technology Institute (IMF), German Aerospace Center (DLR) \\ Munich, Germany (yilei.shi@tum.de)
}

\begin{abstract}
Building footprint information is an essential ingredient for 3-D reconstruction of urban models. The automatic generation of building footprints from satellite images presents a considerable challenge due to the complexity of building shapes. In this work, we have proposed improved generative adversarial networks (GANs) for the automatic generation of building footprints from satellite images. We used a conditional GAN with a cost function derived from the Wasserstein distance and added a gradient penalty term. The achieved results indicated that the proposed method can significantly improve the quality of building footprint generation compared to conditional generative adversarial networks, the U-Net, and other networks. In addition, our method nearly removes all hyperparameter tuning.

Index Terms-building footprint, generative adversarial networks (GANs), conditional generative adversarial networks (CGANs), Wasserstein generative adversarial networks (WGANs)
\end{abstract}

\section{INTRODUCTION}

Building footprint generation is of great importance to urban planning and monitoring, land use analysis, and disaster management. High-resolution satellite imagery, which can provide more abundant detailed ground information, has become a major data source for building footprint generation. Due to the variety and complexity of buildings, building footprint requires significant time and high costs to generate manually (see Fig. 1). As a result, the automatic generation of a building footprint not only minimizes the human role in producing large-scale maps but also greatly reduces time and costs.

Previous studies focusing on building footprint generation can be categorized into four aspects: (a) edge-based, (b) region-based, (c) index-based, and (d) classification-based methods. In edge-based methods, regular shape and line segments of buildings are used as the most distinguishable features for recognition [1]. Region-based methods identify building regions through image segmentation [2]. For indexbased methods, a number of building feature indices are used to describe the characteristics of buildings, which indicate the possible presence of buildings [3]. Classification-based methods, which combine spectral information with spatial features, are among the most widely used approaches, since they can provide more stable and generalized results than the other three methods.

Over the past few years, the most popular and efficient classification approach has been deep learning (DL) [4], which

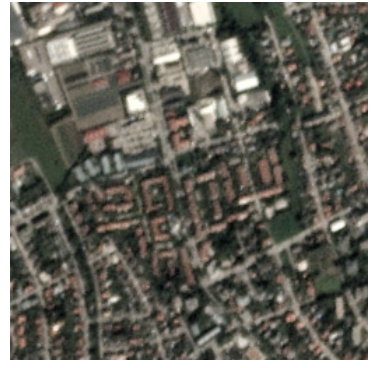

(a)

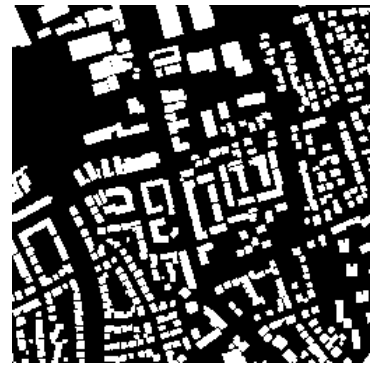

(b)
Fig. 1. (a) Optical imagery of PlanetScope; (b) Building footprint from OSM

has the computational capability for big data. DL methods combine feature extraction and classification and are based on the use of multiple processing layers to learn good feature representation automatically from the input data. Therefore, DL usually possesses better generalization capability, compared to other classification-based methods. In terms of particular DL architectures, several impressive convolutional neural network (CNN) structures, such as ResNet [5] and U-Net [6], have already been widely explored for RS tasks. However, since the goal of CNNs is to learn a parametric translation function by using a dataset of input-output examples, considerable manual efforts are needed for designing effective losses between predicted and ground truth pixels. To address this problem, generative adversarial networks [7] were recently proposed, which learn a mapping from input to output images and tries to classify if the output image is real or fake.

In this regard, one of the motivations of this study was to explore the potential of GANs in building footprint generation by comparing their performance with other $\mathrm{CNN}$ structures. However, GANs also have their own limitations: (a) there is no control over the modes of data being generated, (b) and the training is delicate and unstable. Therefore, several studies have proposed alternatives to traditional GANs, such as conditional GANs [8] and Wasserstein GANs [9]. In order to direct the data generation process and improve the stability of training, we propose combining a conditional GAN, a Wasserstein GAN, and a gradient penalty term for building footprint generation, which are exploited for the first time in the remote-sensing community.

The proposed building footprint generation method is de- 

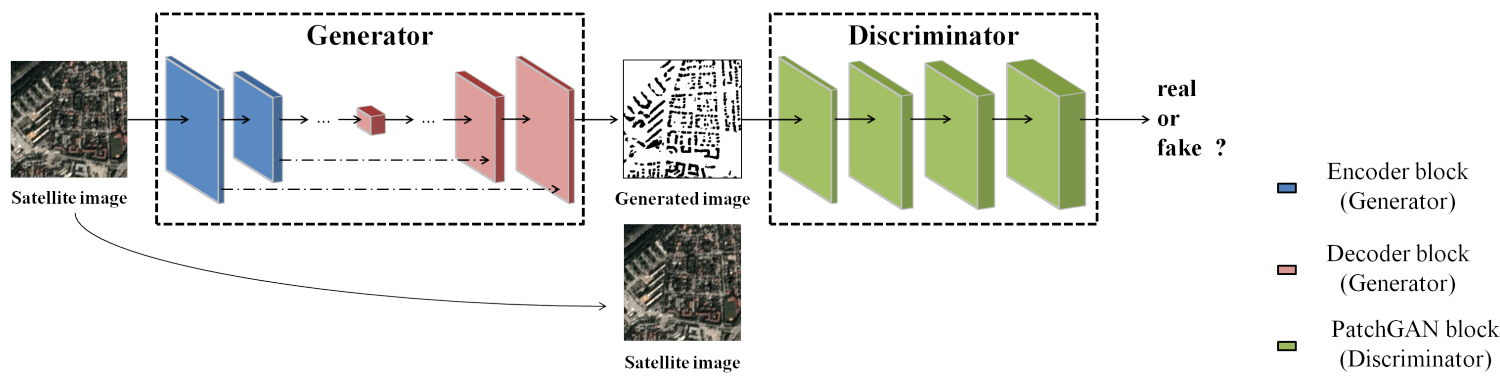

Fig. 2. Network architecture of the proposed method

scribed in Section II. In Section III, the details of the datasets and the experimental results are presented and analyzed. The final conclusions follow in Section IV.

\section{Methodology}

\section{A. Review of GANs}

GANs were firstly proposed in [7] and consist of two neural networks: generator $G$ takes noise variables as input to generate new data instances while discriminator $D$ decides whether each instance of data belongs to the actual training dataset or not. $D$ and $G$ play a two-player minimax game with the objective function as

$$
\mathcal{L}_{G A N}=E_{p_{x}}[\log D(x)]+E_{p_{z}}[\log (1-D(G(z)))]
$$

where $E$ is the empirical estimation of the expected value of the probability. $x$ is the training data with the true data distribution $p_{x}, z$ represents the noise variable sampled from distribution $p_{z}$, and $\bar{x}=G(z)$ represents the generated data instances. $G$ and $D$ are trained simultaneously: for $G$ to minimize $\log (1-D(G(z)))$ and for $D$ to maximize $\log D(x)$.

To address the problem of no control over the modes of data being generated in GANs, Mirza et al. [8] extended GANs to a conditional model, where both the generator and discriminator are conditioned on certain extra information $y$, which could be any kind of auxiliary information, such as class labels. The conditioning is performed by feeding $y$ into both the discriminator and generator as an additional input layer. The objective function of CGANs is constructed as following:

$$
\mathcal{L}_{C G A N}=E_{p_{x}}[\log D(x \mid y)]+E_{p_{z}}[\log (1-D(G(z \mid y)))]
$$

In order to improve the stability of learning of GANs and remove problems like mode collapse, WGANs were proposed by Arjovsky et al. [9], which use an alternative cost function that is derived from an approximation of the Wasserstein distance. They are more likely to provide gradients that are useful for updating the generator than the original GANs.

\section{B. Proposed Method}

In this work, we want to exploit the superiorities of both CGANs and WGANs. Therefore, we propose CWGANs, which can impose a control on the modes of data being generated, and can also achieve more stable training as well. The objective function of CWGANs is given by:

$$
\mathcal{L}_{C W G A N}=E_{p_{x}}[D(x \mid y)]-E_{p_{z}}[D(G(z \mid y))]
$$

However, due to the use of weight clipping in WGANs, CWGANs may still generate low-quality samples or fail to converge in some settings. Therefore, we used an alternative to clipping weights: the addition of a gradient penalty term [10] with respect to its input, whose objective function can be written as:

$$
\mathcal{L}_{G P}=\lambda_{1} E_{p_{x, z}}\left[\left(\|\nabla D(\alpha x+(1-\alpha) G(z \mid y))\|_{2}-1\right)^{2}\right]
$$

where $\lambda_{1}$ is the gradient penalty coefficient, and $\alpha$ is a random number with uniform distribution in $[0,1]$.

In order to let the generator to be located near the ground truth output and to decrease blurring, a traditional loss $L_{1}$ distance is mixed with the CWGAN objective:

$$
\mathcal{L}_{L_{1}}=\lambda_{2} E_{p_{x, z}}\left[\|x-G(z \mid y)\|_{1}\right]
$$

where $\lambda_{2}$ is the coefficient for $L_{1}$ regularization. Finally, our objective function is the combination of CWGAN, gradient penalty term, and $L_{1}$ regularization.

$$
\mathcal{L}=\arg \min _{G} \max _{D} \mathcal{L}_{C W G A N}+\mathcal{L}_{G P}+\mathcal{L}_{L_{1}}
$$

\section{Network Architectures}

The network architecture in this work is shown in Fig. 2. We used the U-Net as the generator architecture. It is an encoder-decoder network with skip connections to concatenate all channels at layer $i$ with those at layer $n-i$, where $n$ is the total number of layers. The Leaky ReLU activation is used for the downsampling process, and the ReLU activation is used for upsampling.

As for the discriminator architecture, the PatchGAN proposed in [11] is exploited to model a high frequency structure. This network tries to classify whether each patch in an image is real or fake. With the discriminator running convolutionally across the image, the ultimate output of $D$ can be provided by averaging all responses.

\section{EXPERIMENTS}

\section{A. Description of Datasets}

In this work, we chose two study areas in Germany, which were Munich and Berlin. We used PlanetScope satellite imagery with three bands (R, G, B) and a spatial resolution of $3 \mathrm{~m}$ to test our proposed method. The corresponding building footprints were downloaded from OpenStreetMap (OSM). We processed the imagery using a $256 \times 256$ sliding window with 
a stride of 75 pixels to produce around 3000 sample patches. The sample patches were divided into two parts, where $70 \%$ were used to train the network and $30 \%$ were used to validate the trained model.

\section{B. Experimental Setup}

The number of both generator and discriminator filters in the first convolution layer was 64 . The downsample factor is 2 in both the discriminator and the encoder of the generator. In the decoder of the generator, deconvolutions were performed with an upsample factor of 2 . All convolutions and deconvolutions had a kernel size of $4 \times 4$, a stride equal to 2 , and a padding size of 1. An Adam solver with a learning rate of 0.0002 was adopted as an optimizer for both networks. Furthermore, we use a batch size of one for each network and trained at 200 epochs. The clipping parameter in CWGAN was 0.01 . For the CWGAN-GP, the gradient penalty coefficient $\lambda_{1}$ was set to 10 as recommended in [10].

\section{Results and Analysis}

In this work, we evaluated the inference performances using metrics for a quantitative comparison: overall accuracy (OA), F1 scores, and IoU scores. The impacts of hyperparameters have been investigated for our proposed methods. Firstly, the influence of different depths $d$ of the U-Net structure has been explored.

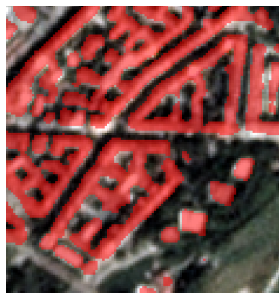

(a)

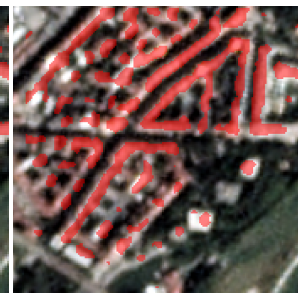

(b)

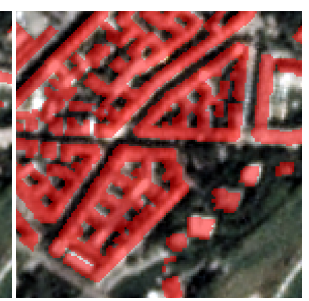

(c)
Fig. 3. Comparison of results generated by U-Net structure with different depths. (a) Depth $(d=5)$; (b) Depth $(d=8)$; (c) Ground truth.

Fig. 3 shows visual results of one patch with different depths compared to the ground truth. As one can see in Fig. 3, a large number of roofs are omitted by the network with $d=8$ but are identified by the depth $d=5$. Similar phenomenas have been reported in [13]. With the network depth increasing, accuracy gets saturated and then degrades rapidly, since adding more layers to a suitably deep model leads to a higher training error. Note that the optimal depth of the network should be comparable with the size of useful features in the imagery in order to achieve high accuracy.

Secondly, we have chosen different coefficients $\left(\lambda_{2}=\right.$ 1,100) of $L_{1}$ loss with the CGAN and CWGAN-GP. The quantitative results are listed in Table II, and results of the sample for visual comparison are in Fig. 4.

When the coefficient of $L_{1}$ loss increased from 1 to 100 , the CGAN results dramatically improved for all evaluation metrics. As one can see from Fig. 4 (a) and (b), the building area generated by the CGAN with $\lambda_{2}=100$ is more correct and complete than that with $\lambda_{2}=1$. Such a result can be potentially explained by the fact that the $L_{1}$ loss term penalizes the distance between ground truth outputs and synthesized outputs, and the synthesized outputs from the $L_{1}$ loss term are better for the training of the discriminator. In contrast, the result of CWGAN-GP with $\lambda_{2}=100$ is slightly better than with $\lambda_{2}=1$, which indicates that our proposed method is not sensitive to hyperparameters. Moreover, it should be noted that the numerical results did not indicate a considerable difference when choosing different hyper-parameter combinations. This is due to the stability of our proposed methods, which nearly removes all hyperparameters tuning and simply uses the default setting.

TABLE I

COMPARISON OF DIFFERENT NETWORKS ON THE TEST DATASETS

\begin{tabular}{cccc}
\hline \hline Methods & Overall Accuracy & F1 score & IoU score \\
\hline CGAN $\left(\lambda_{2}=1\right)$ & $79.42 \%$ & 0.1555 & 0.0842 \\
\hline CGAN $\left(\lambda_{2}=100\right)$ & $85.46 \%$ & 0.5787 & 0.4072 \\
\hline ResNet-DUC & $85.71 \%$ & 0.5881 & 0.4166 \\
\hline U-Net & $86.03 \%$ & 0.6455 & 0.4766 \\
\hline CWGAN & $88.54 \%$ & 0.6737 & 0.5079 \\
\hline CWGAN-GP $\left(\lambda_{2}=1\right)$ & $88.87 \%$ & 0.6821 & 0.5169 \\
\hline CWGAN-GP $\left(\lambda_{2}=100\right)$ & $\mathbf{8 9 . 0 6 \%}$ & $\mathbf{0 . 6 8 3 0}$ & $\mathbf{0 . 5 1 9 4}$ \\
\hline \hline
\end{tabular}

Finally, we applied the selected coefficient of $L_{1}$ loss and depth $(d=5)$ in the generator to our proposed method CWGAN-GP. From Table I, we can see that the proposed method gives the best accuracy for all metrics. Compared to a CGAN, the CWGAN and CWGAN-GP indicate a dramatical increase of segmentation performance. This is because that even when two distributions are located in lower dimensional manifolds without overlaps, the Wasserstein distance can still provide a meaningful representation of the distance in-between. Since the weights in the discriminator of the CWGAN clamped to small values around zero, the parameters of the weights can lie in a compact space, which leads a learning process more stable than that of CGANs. However, a hyperparameter (the size of clipping window) in the CWGAN should still be tuned in order to avoid unstable training. If the clipping window is too large, there will be slow convergence after weight clipping. Moreover, if the clipping window is too small, it will lead to vanishing gradients. Therefore, the proposed CWGAN-GP, which add a gradient penalty term into the loss of discriminator, will improve the stability of the training. The proposed methods (CWGAN and CWGANGP) outperform ResNet-DUC in both numerical results and visual analysis, because the skip connections in generator $G$ combines both the lower and higher layers to generate the final output, retaining more details and better preserving the boundary of the building area. Compared to the U-Net, the proposed methods achieve higher overall accuracy, the F1 score and IoU score, as the min-max game between the 


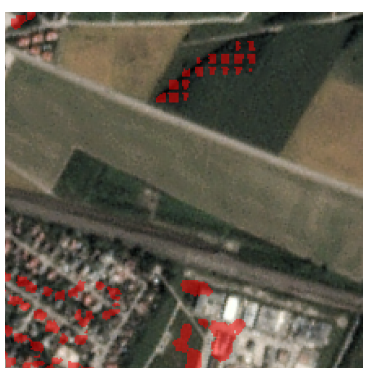

(a)

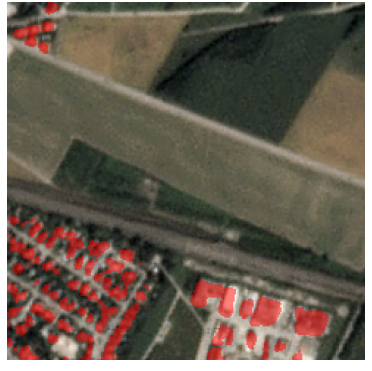

(e)

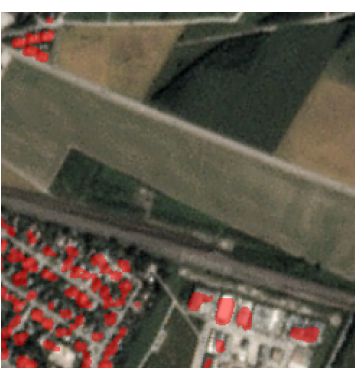

(b)

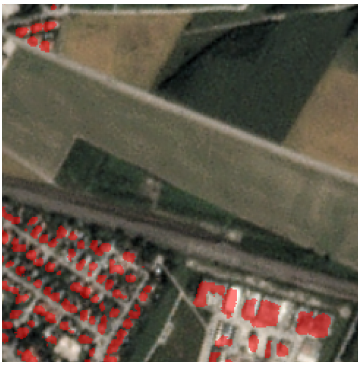

(f)

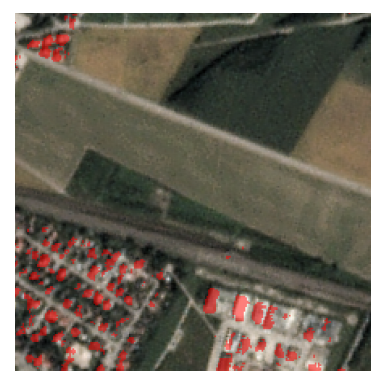

(c)

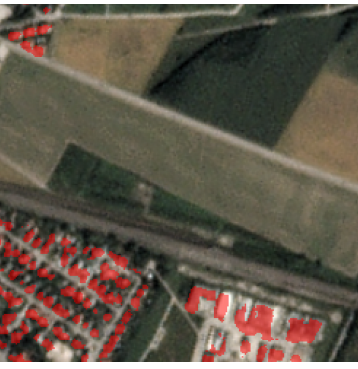

(g)

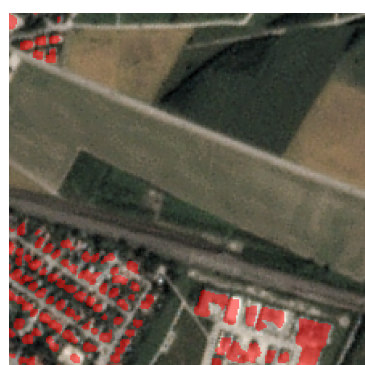

(d)

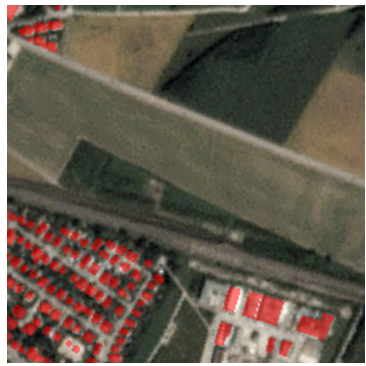

(h)

Fig. 4. Visualized comparison of different networks and coefficients $\lambda_{2}$ of $L_{1}$ loss; (a) CGAN $\left(\lambda_{2}=1\right)$; (b) CGAN $\left(\lambda_{2}=100\right)$; (c) ResNet-DUC; (d) U-Net; (e) CWGAN ( $\left.\lambda_{2}=100\right)$; (f) CWGAN-GP $\left(\lambda_{2}=1\right)$; (g) CWGAN-GP $\left(\lambda_{2}=100\right)$; (h) ground truth.

generator and discriminator of the GAN, motivates both to improve their functionalities.

\section{Conclusion}

GANs, which have recently been proposed, provide a way to learn deep representations without extensively annotated training data. This research aimed to explore the potential of GANs in the performance of building footprint generation and improve its accuracy by modifying the objective function. Specifically, we proposed two novel network architectures (CWGAN and CWGAN-GP) that integrate CGAN and WGAN, as well as a gradient penalty term, which can direct the data generation process and improve the stability of training. PlanetScope satellite imagery of Munich and Berlin was investigated to evaluate the capability of the proposed approaches. The experimental results confirm that the proposed methods can significantly improve the quality of building footprint generation compared to existing networks.

\section{ACKNOWLEDGMENT}

This work is supported by the European Research Council (ERC) under the European Union's Horizon 2020 research and innovation programme (no. ERC-2016-StG-714087, acronym: So2Sat, www.so2sat.eu), the Helmholtz Association under the framework of the Young Investigators Group "SiPEO" (VHNG-1018, www.sipeo.bgu.tum.de), Munich Aerospace e.V. Fakultät für Luft- und Raumfahrt, and the Bavaria California Technology Center (Project: Large-Scale Problems in Earth Observation). The authors thank Planet provide the datasets.

\section{REFERENCES}

[1] J. Wang, X. Yang, X. Qin, X. Ye and Q. Qin, "An efficient approach for automatic rectangular building extraction from very high resolution optical satellite imagery," IEEE Geosci. Remote Sensing Lett., vol. 12, no. 3, pp. 487-491, 2015.

[2] A. O. Ok, "Automated detection of buildings from single VHR multispectral images using shadow information and graph cuts," ISPRS J. Photogramm Remote Sens., vol. 86, pp. 21-40, 2013.

[3] X. Huang and L. Zhang, "A multidirectional and multiscale morphological index for automatic building extraction from multispectral GeoEye-1 imagery," Photogramm. Eng. Remote Sens., vol. 77, no. 7, pp. 721-732, 2011.

[4] X. X. Zhu, D. Tuia, L. Mou, G. Xia, L. Zhang, F. Xu, and F. Fraundorfer, "Deep learning in remote sensing: a comprehensive review and list of resources," IEEE Geosci. Remote Mag., vol. 5, no. 4, pp. 8-36, 2017.

[5] K. He, X. Zhang, S. Ren and J. Sun, "Deep residual learning for image recognition," in Proc. IEEE CVPR, Las Vegas, CA, USA, 2016, pp. 770-778.

[6] O. Ronneberger, P. Fischer and T. Brox, "U-Net: Convolutional networks for biomedical image segmentation," in Proc. MICCAI, 2015, pp. 234 241.

[7] I. Goodfellow et al., "Generative adversarial nets," in Proc. Adv. Neural Inf. Process. Syst., 2014, pp. 2672-2680.

[8] M. Mirza and S. Osindero, "Conditional generative adversarial nets," CoRR, abs/1411.1784, 2014.

[9] M. Arjovsky, S. Chintala and L. Bottou, "Wasserstein GAN," in Proc. ICML, 2017, pp. 214-223.

[10] I. Gulrajani, F. Ahmed, M. Arjovsky, V. Dumoulin and A.C. Courville, "Improved training of Wasserstein GANs," in Proc. Adv. Neural Inf. Process. Syst., 2017, pp. 5679-5779.

[11] P. Isola, J.-Y. Zhu, T. Zhou and A. A. Efros, "Image-to-image translation with conditional adversarial networks," in Proc. IEEE CVPR, Honolulu, Hawaii, USA, 2017, pp. 1125-1134.

[12] P. Wang, P. Chen, Y. Yuan, D. Liu, Ding, Z. Huang, X. Hou, Xiaodi and G. Cottrell, "Understanding Convolution for Semantic Segmentation," CoRR, abs/1702.08502, 2017.

[13] K. He and J. Sun. "Convolutional neural networks at constrained time cost," in Proc. IEEE CVPR, Boston, Massachusetts, USA, 2015, pp. 5353-5360. 